\title{
Poorly controlled ambulatory blood pressure in outpatients with peripheral arterial disease
}

\author{
Nina Dahle ${ }^{a, b}$, Emma Skau ${ }^{c, d}$, Jerzy Leppert ${ }^{c}$, Johan Ärnlöv ${ }^{e, f}$ and Pär Hedberg ${ }^{c, g}$ \\ a'Centre for Clinical Research, Uppsala University, Falun, Sweden; 'PPrimary Health Care Center Britsarvet-Grycksbo, County of Dalarna, \\ Falun, Sweden; 'Centre for Clinical Research, Uppsala University, Västmanland County Hospital, Västerås, Sweden; 'Department of \\ Cardiology, Danderyd University Hospital, Stockholm, Sweden; 'Division of Family Medicine and Primary Care, Department of \\ Neurobiology, Care Sciences and Society, Karolinska Institutet, Huddinge, Sweden; 'SChool of Health and Social Studies, Dalarna \\ University, Falun, Sweden; '9Department of Clinical Physiology, Västmanland County Hospital, Västerås, Sweden
}

\begin{abstract}
Background: Patients with peripheral arterial disease (PAD) are generally less intensively managed than patients with coronary heart disease (CHD), despite that their risk of complications is believed to be equivalent. Identification of PAD patients at risk of poorly controlled blood pressure (BP) could lead to improved treatment, thus lowering the risk of cardiovascular (CV) complications. We aimed to describe the prevalence of poorly controlled cardiovascular (CV) risk factors, focusing on BP, in outpatients with PAD diagnosed in a vascular ultrasound laboratory.

Methods: Consecutive outpatients with carotid and/or lower extremity PAD were included $(n=402)$ and examined with blood sampling, clinical BP, and 24-h ambulatory BP measurements. A poorly controlled clinical BP was defined as $\geq 140 / 90 \mathrm{mmHg}$, ambulatory BP $\geq 130 / 80 \mathrm{mmHg}$, low-density lipoprotein (LDL)-cholesterol level $\geq 2.5 \mathrm{mmol} / \mathrm{L}$, and glycated hemoglobin ( $\mathrm{HbA} 1 \mathrm{c}$ ) level $>53 \mathrm{mmol} / \mathrm{mol}$ in those with diabetes.

Results: Most of the patients had poorly controlled clinical (76.6\%) and ambulatory BP (51.7\%) profiles. Antihypertensive medications were prescribed in $84 \%$ of the patients. However, $>40 \%$ of them used only 0-1 medication, and $<25 \%$ of them used three or more agents. Clinical BP, a low number of medications, body mass index, and the presence of diabetes independently predicted a poorly controlled ambulatory BP. Nearly one-third of the patients were smokers, and most of the cohort had an LDL-cholesterol level of $\geq 2.5 \mathrm{mmol} / \mathrm{L}$. An $\mathrm{HbA} 1 \mathrm{c}$ level of $>53 \mathrm{mmol} / \mathrm{mol}$ was present in $55 \%$ of diabetic patients.

Conclusion: Poorly controlled clinical and ambulatory systolic BP profiles were common. In addition, suboptimal control of other important CV risk factors was detected. The findings of this study highlight the need for better preventive efforts against CV risk factors in outpatients with PAD.
\end{abstract}

\section{ARTICLE HISTORY}

Received: 13 February 2021

Revised: 29 March 2021

Accepted: 29 March 2021

Published: 29 April 2021

\section{KEYWORDS}

Carotid artery disease; cardiovascular risk factors; hypertension; smoking; hyperlipidemia; preventive efforts

\section{Introduction}

Peripheral arterial disease (PAD) is a common clinical manifestation of systemic arterial atherosclerosis, affecting arteries other than the coronary arteries, intracranial arteries, and the aorta $(1,2)$. Atherosclerosis is often generalized, and many affected patients also have coronary artery disease (CAD) (2). Patients can present with a spectrum of symptoms, such as claudication, but most are asymptomatic, and all face a substantially increased risk of major cardiovascular (CV) events and deaths $(3,4)$. PAD is the third leading cause of CV morbidity, after CAD and stroke (5). It is considered to be equivalent to the coronary heart disease risk, warranting aggressive secondary prevention against CV risk factors. However, patients with PAD are generally less intensively managed compared with those with CAD $(1,6-9)$.
The overall prevalence of hypertension in adults is about $30-45 \%$, and it becomes more common with advancing age (10). Among registered diagnoses in hospital care and primary health care in Sweden, hypertension is the diagnosis with the highest prevalence $(11,12)$. Antihypertensive treatments are well established, safe, and highly effective (13). However, many patients with hypertension have inadequate control of their BP or undergo no treatment at all (10). Some of the factors found to be associated with poorly controlled BP in the general population include diabetes, older age, obesity, multi-drug regimens, lack of information on hypertension, and living alone (14-18). There is growing evidence that ambulatory BP measurement (ABPM) is a strong predictor of organ damage and $C V$ outcomes. It can provide important clinical information beyond clinical BP measurements, such as revealing nocturnal 
dipping patterns (19-25). However, how ambulatory BP is controlled and how ABPM can be useful in patients with PAD is not well studied. To further identify patients with PAD at risk of poor BP control could lead to individualized and improved BP treatments, and thereby a lower risk for CV complications (15). In this study, we aimed to describe the prevalence of poorly controlled CV risk factors, focusing on ambulatory BP in consecutive outpatients with PAD diagnosed in a vascular ultrasound laboratory.

\section{Methods}

\section{Study population}

Analyses were based on patients included in the Peripheral Arterial Disease in Västmanland (PADVa) study (26). All patients visiting the ultrasound laboratory of the Department of Vascular Surgery at the Västmanland County Hospital in Västerås, Sweden, from April 2006 to February 2011, were considered for inclusion. Reasons for referral include claudication (45\%), transient ischemic attack or stroke (26\%), aortic aneurysm (8\%), heart murmur (5\%), suspected renal artery stenosis or renovascular hypertension (4\%), and others (12\%). Every patient was examined with ultrasonography to identify any stenosis in the internal carotid artery (ICA). Patients with symptoms of claudication also underwent ankle BP measurement to calculate the ankle-brachial index $(A B I)$ and ultrasonography of the arteries in the symptomatic leg. The patients were invited to participate in the PADVa study if they met at least one of the following inclusion criteria: 1) mild to severe stenosis or occlusion of the ICA, 2) symptoms of claudication combined with $A B I \leq 0.90$ in the symptomatic lower extremity, or 3) symptoms of claudication combined with ultrasonographic evidence of arterial occlusive disease in the same extremity.

In total, 452 patients (73.6\%) accepted the invitation to join the study. Everyone in the study was offered ABPM, of whom 35 individuals refused. We excluded patients with $<10$ daytime or $<5$ night-time ABPM readings ( $n=15$ ) (27), leaving 402 patients for analysis.

The study was approved by the Ethics Committee of Uppsala University, Sweden (Dnr 2005:382). All participants gave their written informed consent to participate. The study is registered with ClinicalTrials.gov number NCT01452165.

\section{Examination protocol}

All patients were invited to the Department of Clinical Physiology and were examined according to a standard examination protocol, comprising a questionnaire, including the number of persons in household (living alone vs. cohabitating), educational level (low level was defined as primary school or less), smoking status (smoking defined as regular smoking within the past year), medical history, medication, and physical activity (physically inactive was defined as mostly sedentary with more demanding activities, such as walking, biking, gardening $<2 \mathrm{~h}$ per week). Self- reported diagnoses of CV disease and diabetes mellitus were confirmed from the medical records.

Participants fasted overnight, and venous blood samples were taken by trained staff and immediately sent to the accredited Laboratory of Clinical Chemistry, Västmanland County Hospital, Västerås. The estimated glomerular filtration rate (eGFR) was calculated from creatinine levels standardized by isotope dilution mass spectrometry (SYNCHRON LX or UniCel DxC instruments; Beckman Coulter, Inc., Brea, CA, USA) using the Chronic Kidney Disease Epidemiology Collaboration (CKD-EPI) formula (28).

Glycated hemoglobin (HbA1c) mono was determined using a TOSOH Glycohemoglobin Analyzer G7 (Tosoh, Japan) and calibrated against the Swedish Mono-S method. The HbA1c was calculated from $\mathrm{HbA} 1 \mathrm{c}$ mono using the formula: $10.45 \times(\mathrm{HbA} 1 \mathrm{c}$ mono level) - 10.62 (International Federation of Clinical Chemistry and Laboratory Medicine, IFCC standard). The treatment target level for diabetics ( $\leq 53 \mathrm{mmol} / \mathrm{mol}$ ) was based on the European Society of Cardiology (ESC) guidelines from 2003 (29).

The serum total cholesterol (TC) concentration was determined using a UniCel DxC 800 or SYNCHRON LX20 Analyzer (Beckman Coulter, Inc.). Levels of low-density lipoprotein cholesterol (LDL-cholesterol) were calculated from those of TC, high-density lipoprotein cholesterol (HDL-cholesterol), and triglycerides using the Friedewald equation: (LDL-cholesterol = TC - HDL-cholesterol - Triglyceride levels $\times 0.45$ ). The treatment target levels of LDL-cholesterol are based on the ESC guidelines from 2003 (<2.5 mmol/L) and 2019 (<1.4 mmol/L) (29, 30).

Based on a standard 12-lead surface electrocardiography (ECG), left ventricular (LV) hypertrophy was defined as the Sokolow-Lyon voltage $>35 \mathrm{mV}$ or Cornell voltage $>28 \mathrm{mV}$ in men and $>20 \mathrm{mV}$ in women.

\section{Ankle blood pressure and carotid ultrasound}

Blood pressure in both arms and ankles was measured in all included participants in a supine position after at least 5 min rest. The ankle BP was measured in the bilateral dorsalis pedis and posterior tibial arteries using an inflatable leg-cuff, an aneroid sphygmomanometer, and a handheld Doppler instrument with a $5-\mathrm{MHz}$ probe. The $\mathrm{ABI}$ was calculated by dividing the highest ankle pressure by the highest BP of both arms. An abnormal ABI was defined as $\leq 0.90$ or $\geq 1.40$ in either leg.

Carotid artery ultrasonography has been described in detail (26). Briefly, grading of ICA lesions into normal artery, plaque without flow disturbance, mild/moderate/severe stenosis, or occlusion was based on gray-scale images, color flow Doppler scans, and spectral Doppler blood flow velocities.

\section{Clinical and ambulatory BPs}

Clinical BP was measured manually by trained technicians and was obtained from the non-dominant arm or from the other arm if the systolic BP was $>10 \mathrm{mmHg}$ higher. The BP was measured from participants in the supine position after at least 5 min rest 
and was rounded up to the nearest $2 \mathrm{mmHg}$. Using the arm from which clinical BP was obtained, the ABPM 04 instrument (Meditech Ltd., Budapest, Hungary) was applied for 24-h ABPM with readings taken every 20 min (31). Three different cuff-sizes were available and selected depending on the size of the patient's upper arm. Day- and night-time periods were assessed from the time of awakening and sleeping entered by the patient in a diary card.

The clinical BP was defined as poorly controlled if systolic $B P$ was $\geq 140 \mathrm{mmHg}$ or diastolic $B P$ was $\geq 90 \mathrm{mmHg}$. The corresponding definition for ambulatory BP was a 24-h ambulatory systolic BP of $\geq 130 \mathrm{mmHg}$ or diastolic BP $\geq 80$ $\mathrm{mmHg}(10,32)$. White coat hypertension was defined as a poorly controlled clinical BP combined with a well-controlled ambulatory BP, whereas masked hypertension was defined as the reverse condition, that is, a well-controlled clinical BP in combination with a poorly controlled ambulatory BP (20).

\section{Statistics}

Data are presented as mean \pm standard deviation or frequency and (percentage). To investigate potential predictors of a poorly controlled ambulatory $\mathrm{BP}$, we used logistic regression analysis. In a multivariable model, we included available variables that have been proposed or established in previous studies: age, sex, body mass index (BMI), smoking habit, educational level, living alone, physical activity, diabetes, eGFR, previous myocardial infarction (MI), stroke, heart failure, abnormal $A B I$, ICA stenosis, number of antihypertensive medications, LV hypertrophy, and clinical systolic and diastolic BP (14-18). Statistical analyses were performed using R 3.5.3 (R Foundation for Statistical Computing, 2019, Vienna, Austria; http://www.rproject.org). Two-sided $P$ values $<0.05$ were considered to be statistically significant

\section{Results}

\section{Baseline characteristics}

Nearly a third of the patients (28\%) were smokers (Table 1). Among patients with a poorly controlled ambulatory BP, diabetes mellitus was more frequent (29\%) compared with the those with well-controlled ambulatory BP (19\%). ICA stenosis

Table 1. Characteristics of patients with peripheral arterial disease, overall and stratified by well-controlled $(<130 / 80 \mathrm{mmHg})$ and poorly controlled ( $\geq 130 / 80 \mathrm{mmHg}$ ) ambulatory 24-h blood pressure (BP).

\begin{tabular}{|c|c|c|c|}
\hline & $\begin{array}{l}\text { All patients; } \\
\quad n=402\end{array}$ & $\begin{array}{l}\text { Well-controlled ambulatory } \\
\text { (Amb) BP; } n=194\end{array}$ & $\begin{array}{l}\text { Poorly controlled } \\
\text { Amb BP; } n=208\end{array}$ \\
\hline Male (sex) & $240(59.7 \%)$ & $118(60.8 \%)$ & $122(58.7 \%)$ \\
\hline Smoking & $112(28.1 \%)$ & $55(28.9 \%)$ & $57(27.4 \%)$ \\
\hline Low education level & $229(57.0 \%)$ & $116(59.8 \%)$ & $113(54.3 \%)$ \\
\hline Living alone & $105(26.2 \%)$ & $58(29.9 \%)$ & $47(22.8 \%)$ \\
\hline Diabetes mellitus & $97(24.1 \%)$ & $37(19.1 \%)$ & $60(28.8 \%)$ \\
\hline Abnormal ankle-brachial index & $225(56.0 \%)$ & $109(56.2 \%)$ & $116(55.8 \%)$ \\
\hline Internal carotid artery stenosis & $303(75.4 \%)$ & $139(71.6 \%)$ & $164(78.8 \%)$ \\
\hline Previous myocardial infarction & $78(19.4 \%)$ & $39(20.1 \%)$ & $39(18.8 \%)$ \\
\hline Previous stroke & $42(10.4 \%)$ & $22(11.3 \%)$ & $20(9.6 \%)$ \\
\hline LDL cholesterol $\geq 1.4 \mathrm{mmol} / \mathrm{L}$ & $385(97.7 \%)$ & $185(96.9 \%)$ & $200(98.5 \%)$ \\
\hline Estimated glomerular filtration rate $\left(\mathrm{mL} / \mathrm{min} / 1.73 \mathrm{~m}^{2}\right)$ & $73.9 \pm 17.5$ & $73.8 \pm 16.4$ & $73.9 \pm 18.4$ \\
\hline Glycated hemoglobin $(\mathrm{mmol} / \mathrm{mol})$ & $42.3 \pm 10.3$ & $41.0 \pm 8.3$ & $43.4 \pm 11.8$ \\
\hline Left ventricular hypertrophy & $66(16.7 \%)$ & $23(12.0 \%)$ & $43(21.0 \%)$ \\
\hline Angiotensin-converting enzyme inhibitor/Angiotensin receptor blocker & $230(57.2 \%)$ & $108(55.7 \%)$ & $122(58.7 \%)$ \\
\hline Betablockers & $200(49.8 \%)$ & $103(53.1 \%)$ & $97(46.6 \%)$ \\
\hline Diuretics & $96(23.9 \%)$ & $50(25.8 \%)$ & $46(22.1 \%)$ \\
\hline Calcium inhibitor & $145(36.1 \%)$ & $64(33.0 \%)$ & $81(38.9 \%)$ \\
\hline Statins & $329(81.8 \%)$ & $161(83.0 \%)$ & $168(80.8 \%)$ \\
\hline \multicolumn{4}{|l|}{ Blood pressure } \\
\hline Clinical systolic blood pressure (SBP) (mmHg) & $153 \pm 21$ & $143 \pm 17.7$ & $162 \pm 19.7$ \\
\hline
\end{tabular}

Values are presented as mean $\pm S D$ or frequency (percentage). There were missing values in the following categories: smoking $(n=4)$, living alone $(n=2)$, heart failure $(n=1)$, total cholesterol $(n=2)$, LDL cholesterol $(n=8)$, eGFR $(n=1)$, and LV hypertrophy $(n=6)$. 
and LV hypertrophy were more prevalent among patients with poorly controlled ambulatory BP. Most of the patients (53\%) had an LDL-cholesterol level of $\geq 2.5 \mathrm{mmol} / \mathrm{L}$, and almost all reported a level of $\geq 1.4 \mathrm{mmol} / \mathrm{L}$. Of the 97 patients with diabetes mellitus, 53 patients $(55 \%)$ had an $\mathrm{HbA} 1 \mathrm{c}$ level of $>53 \mathrm{mmol} / \mathrm{mol}$, whereas this was seen only in six (2\%) of the 305 patients without documented diabetes.

\section{Clinical and ambulatory BPS}

Most of our patients with PAD had poorly controlled BPs, especially clinical BP (76.6\%), but also ambulatory BP $(51.7 \%)$ (Figure 1). A clinical systolic BP of $\geq 140 \mathrm{mmHg}$ was found in 307 (76.4\%) patients, and a clinical diastolic BP of $\geq 90 \mathrm{mmHg}$ was observed in $58(14.4 \%)$ patients. If a poorly controlled clinical BP was re-defined as systolic BP $>140 \mathrm{mmHg}$ or diastolic BP $>90 \mathrm{mmHg}$, the prevalence rate was found to be $70.4 \%$ ( $n=283$ ). Results of ABPM revealed that 120 (29.9\%) patients exhibited white coat hypertension and 20 (5.0\%) patients showed masked hypertension. An ambulatory 24-h systolic BP of $\geq 130 \mathrm{mmHg}$ was detected in 207 (51.5\%) patients and a 24-h diastolic BP of $\geq 80 \mathrm{mmHg}$ observed in 40 (10.0\%).

Antihypertensive medications were prescribed in $84 \%$ of patients. The number of drugs was remarkably similar in patients with well-controlled and poorly controlled ambulatory BP (Figure 2). More than $40 \%$ of the patients were taking only $0-1$ medication, while less than $25 \%$ of patients were using three or more agents.

\section{Predictors of poorly controlled ambulatory BP}

In a logistic regression analysis, higher clinical systolic BP, higher BMI, fewer antihypertensive medications, and diabetes mellitus were independent predictors of a poorly controlled ambulatory BP independently (Table 2). Clinical diastolic BP was not associated with poorly controlled ambulatory BP. In Figure 3, the probability of poorly controlled ambulatory BP is illustrated depending on the diabetic status and levels of clinical systolic BP and of BMI after adjustment for the other variables listed in Table 2. Patients with diabetes mellitus had similar clinical

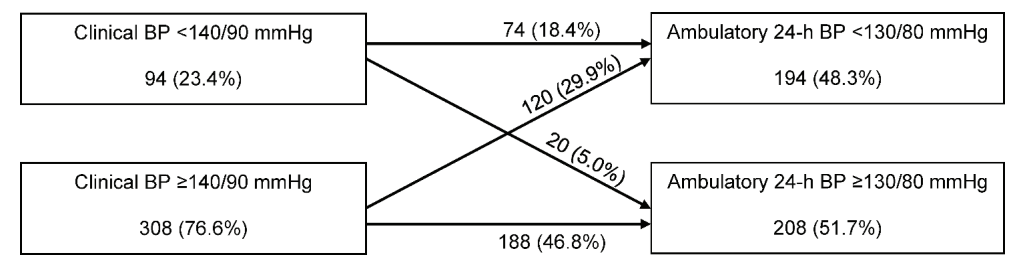

Figure 1. Flowchart showing the prevalence of well and poorly controlled levels of clinical and ambulatory 24-h blood pressure (BP) in 402 outpatients with peripheral arterial disease.

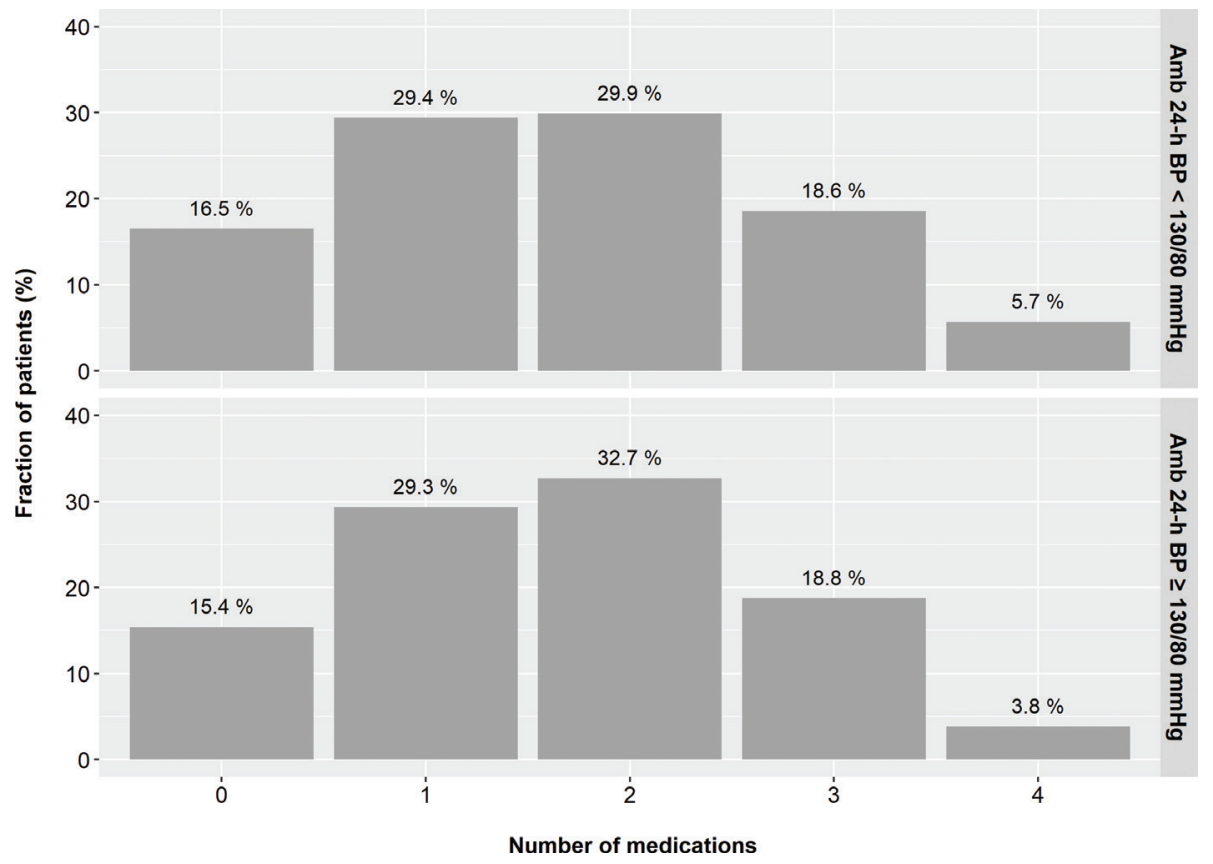

Figure 2. The distribution of the number of prescribed antihypertensive medications according to ambulatory blood pressure levels. 
systolic BP profiles compared with those without diabetes $(154 \pm 20$ vs. $153 \pm 21 \mathrm{mmHg} ; P=0.604)$ but a higher ambulatory 24-h systolic BP (135 \pm 15 vs. $129 \pm 13 \mathrm{mmHg} ; P=0.002)$.

Table 2. Clinical characteristics independently associated with a poorly controlled 24 -h ambulatory blood pressure (i.e. $\geq 130 / 80 \mathrm{mmHg}$ ) in patients with peripheral arterial disease.

\begin{tabular}{|c|c|c|}
\hline & Odds ratio $(95 \% \mathrm{Cl})$ & $p$ \\
\hline Age (for every 10-year increase) & $0.79(0.52-1.21)$ & 0.281 \\
\hline Male (sex) & $1.00(0.60-1.66)$ & 0.998 \\
\hline Body mass index & 1.09 (1.02-1.17) & 0.009 \\
\hline Smoking & $1.23(0.68-2.23)$ & 0.486 \\
\hline Low education & $0.79(0.48-1.31)$ & 0.370 \\
\hline Living alone & $0.81(0.47-1.42)$ & 0.464 \\
\hline Physically inactive & $1.04(0.60-1.80)$ & 0.900 \\
\hline Diabetes & $2.03(1.10-3.75)$ & 0.024 \\
\hline $\begin{array}{l}\text { Estimated glomerular filtration rate } \\
\text { (for every } 10 \text {-unit increase) }\end{array}$ & $0.94(0.80-1.10)$ & 0.412 \\
\hline Previous myocardial infarction & $1.18(0.60-2.33)$ & 0.632 \\
\hline Stroke & $0.79(0.35-1.78)$ & 0.563 \\
\hline Heart failure & $0.35(0.11-1.11)$ & 0.074 \\
\hline Abnormal ankle-brachial index & $1.11(0.64-1.93)$ & 0.708 \\
\hline Internal carotid artery stenosis & $1.44(0.77-2.69)$ & 0.251 \\
\hline Number of medications & $0.67(0.52-0.86)$ & 0.001 \\
\hline Left ventricular hypertrophy & $1.87(0.95-3.68)$ & 0.071 \\
\hline $\begin{array}{l}\text { Clinical systolic blood pressure } \\
\text { (for every } 10 \text {-unit increase) }\end{array}$ & $1.81(1.52-2.15)$ & $<0.001$ \\
\hline $\begin{array}{l}\text { Clinical diastolic blood pressure } \\
\text { (for every } 10 \text {-unit increase) }\end{array}$ & $1.00(0.74-1.35)$ & 0.998 \\
\hline
\end{tabular}

Values are odds ratios and $95 \%$ confidence intervals $(\mathrm{Cl})$ for every one unit increase in continuous independent variables, unless stated otherwise. Analysis is based on 388 patients in a multivariable logistic regression model.

\section{Discussion}

We found that both clinical and ambulatory systolic BPs were poorly controlled in our outpatients with PAD, and that many of these patients appeared to have undergone suboptimal treatment with few antihypertensive medications. Patients with diabetes had an increased risk of poorly controlled ambulatory BP. Moreover, a high proportion of the patients were still smokers and with hyperlipidemia, further emphasizing that preventive care against CV risk factors could be improved in such patients. Among the patients with poorly controlled clinical BP, one-third had white coat hypertension, whereas only $5 \%$ of the patients exhibited masked hypertension. BMI, diabetes mellitus, number of medications, and clinical systolic BP were independent predictors of a poorly controlled ambulatory BP.

The results of this study suggest that a substantial proportion of outpatients with PAD have undertreated systolic hypertension. In contrast, diastolic BPs were better controlled, which is in accordance with previous findings in patients with PAD and likely derives from the stiffness of arteries caused by atherosclerosis (32).

Elevated BP is the most important risk factor for death and disability worldwide, accounting for almost 10 million deaths in 2015 (13). Only a few previous studies have investigated ambulatory BP levels in patients with PAD. Skoglund et al. evaluated clinical and ambulatory BP profiles in 98 male patients with lower extremity arterial disease (33). Compared with their findings, our patients had similar mean clinical systolic and diastolic BPs (153/77 vs. 151/79 mmHg), whereas the mean ambulatory 24-h BPs were lower in our population

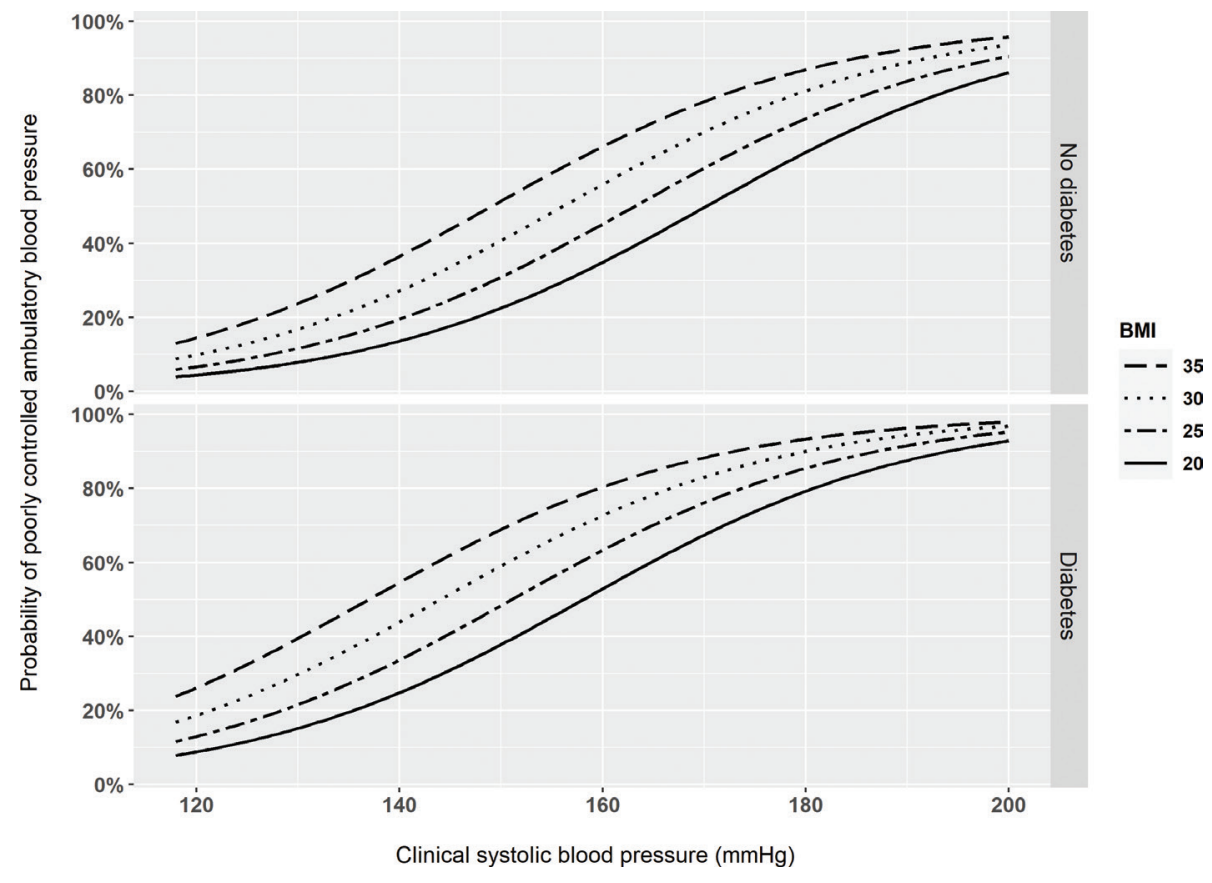

Figure 3. Probability of poorly controlled ambulatory 24 -h blood pressure (i.e. $\geq 130 / 80 \mathrm{mmHg}$ ) according to the clinical systolic blood pressure, body mass index (BMI) in kg/m², and occurrence of diabetes mellitus. The probabilities are adjusted for the variables shown in Table 2. 
(131/68 vs. $142 / 78 \mathrm{mmHg})$. This might have been because of a higher rate of antihypertensive medications ( $84 \%$ vs. $70 \%$ ) in our population.

Data on optimal BP treatment target levels in patients with PAD are conflicting. Possibly, lowering BP too far in these patients might reduce perfusion in lower limbs and increase the risk of PAD-related events. In a reanalysis of data from the Antihypertensive and Lipid-Lowering Treatment to Prevent Heart Attack Trial (ALLHAT), the association of clinical BPs with incident PAD events (hospitalization, procedures, medication, or death related to PAD) was evaluated. The authors found a U-shaped association of systolic BP with PAD events. Higher rates of lower extremity PAD events were observed with high $(>160 \mathrm{mmHg})$ as well as with low $(<120 \mathrm{mmHg})$ systolic BP and with low diastolic BP (<70 mmHg) (34). In contrast, a metaanalysis of small prospective studies suggested that antihypertensive treatment was not associated with worsening symptoms or outcomes in PAD, and there was a trend toward improvement in leg ischemia (35). Although optimal BP target levels specific for PAD are unclear, the evidence so far has not been strong enough to modify guidelines for patients with PAD (34). The suboptimal treatment of hypertension remains worrying, because patients with PAD are at high risk of CVrelated mortality $(9,36)$ but are less intensively treated and have a higher CV mortality than those with MI (37).

A combination of drugs is recommended as first-line therapy against hypertension for most patients according to the latest guidelines (10). In a recent Swedish nationwide registry study of long-term prophylactic treatment patterns in PAD, $60 \%$ of the patients had any antihypertensive treatment (8), which is considerably lower than the $84 \%$ in our study population. Nonetheless, there was a high prevalence of poorly controlled hypertension in our patient population, and more than $40 \%$ of patients were using only one or no BPlowering agents.

In the general population, white coat hypertension can be found in up to $30-40 \%$ of subjects with an elevated in-clinic BP level, and masked hypertension can be observed in around $15 \%$ of patients with a normal in-clinic BP level (10). Ambulatory BP in our patients revealed fewer cases with masked hypertension (5\%) and a similar proportion with white coat hypertension (30\%). These findings suggest that ABPM is of minor importance in detecting masked hypertension in patients with PAD. Instead, ABPM may be more useful in detecting white coat hypertension when attempts to reduce clinical BPs fail.

The clinical features that predicted a high ambulatory BP were not surprising. The prevalence of hypertension is known to be increased in individuals with other CV risk factors, such as obesity and diabetes $(30,31)$. That high clinical BP increases the risk of poorly controlled ambulatory $\mathrm{BP}$ and that the use of more hypertensive medications reduces $\mathrm{BP}$, was also expected.

In parallel with poorly controlled BP profiles, it is obvious that the control of other important CV risk factors was weak in our population. Nearly $30 \%$ of our patients were smokers, which is markedly more common than in the general population, where the prevalence of smoking in Sweden has decreased from 14\% to $7 \%$ between 2006 and 2018 according to the Public Health Agency of Sweden. Smoking is a particularly strong risk factor for PAD (1), and smoking duration seems to be a risk factor for women after only 10 years of smoking (36). Smoking cessation is highly beneficial and should be prioritized to reduce CV events and mortality rates (2).

The latest European guidelines recommend lipid-lowering treatments in all patients with PAD, including a maximum tolerated dose of statins, plus ezetimibe or in combination with a proprotein convertase subtilisin/kexin type 9 (PCSK9) inhibitor if needed. The use of statins and addition of a PCSK9 inhibitor to further lower the LDL-cholesterol level seem to provide a favorable effect on lower limb prognosis, in addition to further reduction of CV events $(38,39)$. A nationwide Swedish study found that $74 \%$ of patients with Ml used statins, but only $53 \%$ of patients with PAD did so (37). Among our patients, more than $80 \%$ were using statins; however, the majority of these individuals still had excessive levels of LDL-cholesterol (Table 2).

Diabetes mellitus is strongly associated with an elevated risk of PAD and with worse outcomes $(1,10)$. Here, outpatients with PAD and concomitant diabetes had an increased risk of poorly controlled ambulatory BP.

That many of our outpatients with PAD were still smokers and with LDL-cholesterol levels of $\geq 2.5 \mathrm{mmol} / \mathrm{L}$, and that many with diabetes had $\mathrm{HbA} 1 \mathrm{c}$ levels $>53 \mathrm{mmol} / \mathrm{mol}$, in addition to poorly controlled hypertension, suggests the need for improved preventive care in such individuals.

This research study was limited to consecutive outpatients of European origin who were found to have lower extremity and/or carotid artery disease in a visit to a vascular ultrasound laboratory. The invited patients who declined to join the study ( $n=162)$ did not differ in age $(P=0.68)$ or sex $(P=0.93)$ compared with the participants. However, if more burdened with disease, these dropouts may have been a source of bias. The participants who declined ABPM $(n=35)$ or were excluded due to few ABPM readings ( $n=15$ ) did not differ significantly from the included participants regarding age, sex, BMI, smoking, diabetes, previous myocardial infarction, or stroke (all $p$-values $>0.095$ ). Data on medication were based on selfreported information, which might be a cause of informational bias. However, good agreement between patient interview and computerized pharmacy records has been found in the elderly population (40). The clinical BPs were measured on one occasion only. Furthermore, we did not have any information regarding previous efforts to intensify antihypertensive treatments in these patients.

In our outpatients with PAD, diagnosed in a vascular ultrasound laboratory, poorly controlled clinical and ambulatory systolic BP profiles were common. Those receiving a higher number of antihypertensive agents had a better BP control, thus suggesting room for improvement. In addition, we found a suboptimal control of other important CV risk factors in these patients, including smoking, $\mathrm{HbA} 1 \mathrm{c}$ levels in those with diabetes mellitus, and LDL-cholesterol levels. This study will help in motivating intensified preventive efforts against CV complications in outpatients with PAD. 


\section{Acknowledgements}

The authors thank the participants and staff of the PADVa study.

\section{Disclosure statement}

The authors declare no potential conflicts of interest with respect to the research, authorship, and/or publication of this article.

\section{Funding}

The research study was supported by Sparbanksstiftelsen Nya, the County of Västmanland, and the Swedish Medical Association.

\section{Notes on contributors}

Nina Dahle, MD, PhD student in medical sciences at Uppsala University, Sweden. Primary Health Care Center BritsarvetGrycksbo, Falun, Sweden.

Emma Skau, MD, PhD student in medical sciences at Uppsala University, Sweden. Department of Cardiology, Danderyd University Hospital, Stockholm, Sweden.

Jerzy Leppert, MD, Professor, Centre for Clinical Research, Uppsala University, Västerås, Sweden.

Johan Ärnlöv, MD, Professor at the Division of Family Medicine and Primary Care, Department of Neurobiology, Care Sciences and Society, Karolinska Institutet, Huddinge, and School of Health and Social Studies, Dalarna University, Falun, Sweden.

Pär Hedberg, MD, Senior consultant at the Department of Clinical Physiology, Västerås, Sweden. Associate professor at the Centre for Clinical Research, Uppsala University, Västerås, Sweden.

\section{ORCID}

Nina Dahle Dhttps://orcid.org/0000-0003-1444-2515

Emma Skau (Dhttps://orcid.org/0000-0002-5625-1146

Jerzy Leppert (Dhttps://orcid.org/0000-0003-1433-0329

Johan Ärnlöv (Dhttps://orcid.org/0000-0002-6933-4637

Pär Hedberg Dhttps://orcid.org/0000-0001-5731-966X

\section{References}

1. Criqui $\mathrm{MH}, \mathrm{Aboyans} \mathrm{V}$. Epidemiology of peripheral artery disease. Circ Res 2015; 116: 1509-26. doi: 10.1161/CIRCRESAHA.116.303849

2. Aboyans V, Ricco JB, Bartelink MEL, Bjorck M, Brodmann M, Cohnert T, et al. 2017 ESC guidelines on the diagnosis and treatment of peripheral arterial diseases, in collaboration with the European Society for Vascular Surgery (ESVS): document covering atherosclerotic disease of extracranial carotid and vertebral, mesenteric, renal, upper and lower extremity arteries. Endorsed by: the European Stroke Organization (ESO)The Task Force for the Diagnosis and Treatment of Peripheral Arterial Diseases of the European Society of Cardiology (ESC) and of the European Society for Vascular Surgery (ESVS). Eur Heart J 2018; 39: 763-816. doi: 10.1093/ eurheartj/ehx095

3. Feringa HH, Van Waning VH, Bax JJ, Elhendy A, Boersma E, Schouten O, et al. Cardioprotective medication is associated with improved survival in patients with peripheral arterial disease. J Am Coll Cardiol 2006; 47: 1182-7. doi: 10.1016/j.jacc.2005.09.074

4. Shu J, Santulli G. Update on peripheral artery disease: epidemiology and evidence-based facts. Atherosclerosis 2018; 275: 379-81. doi: 10.1016/j. atherosclerosis.2018.05.033

5. Fowkes FG, Murray GD, Butcher I, Heald CL, Lee RJ, Chambless LE, et al. Ankle brachial index combined with Framingham Risk Score to predict cardiovascular events and mortality: a meta-analysis. JAMA 2008; 300: 197-208. doi: 10.1001/jama.300.2.197

6. Lindholt JS, Sogaard R. Population screening and intervention for vascular disease in Danish men (VIVA): a randomised controlled trial. Lancet (London, England) 2017; 390: 2256-65. doi: 10.1016/ S0140-6736(17)32250-X

7. Hirsch AT, Criqui MH, Treat-Jacobson D, Regensteiner JG, Creager MA, Olin JW, et al. Peripheral arterial disease detection, awareness, and treatment in primary care. JAMA 2001; 286: 1317-24. doi: 10.1001/ jama.286.11.1317

8. Sartipy F, Lundin F, Wahlberg E, Sigvant B. Cardiovascular long-term outcome and prophylactic treatment patterns in peripheral arterial disease in a population-based cohort. Eur Heart J Qual Care Clin Outcomes 2019; 5: 310-20. doi: 10.1093/ehjqcco/qcz037

9. Subherwal S, Patel MR, Kober L, Peterson ED, Bhatt DL, Gislason GH, et al. Peripheral artery disease is a coronary heart disease risk equivalent among both men and women: results from a nationwide study. Eur J Prev Cardiol 2015; 22: 317-25. doi: 10.1177/2047487313519344

10. Williams B, Mancia G, Spiering W, Agabiti Rosei E, Azizi M, Burnier M, et al. $2018 \mathrm{ESC} / \mathrm{ESH}$ Guidelines for the management of arterial hypertension: the task force for the management of arterial hypertension of the European Society of Cardiology and the European Society of Hypertension: the task force for the management of arterial hypertension of the European Society of Cardiology and the European Society of Hypertension. J Hypertens 2018; 36: 1953-2041. doi: 10.1097/ HJH.0000000000001940

11. Wirehn AB, Karlsson HM, Carstensen JM. Estimating disease prevalence using a population-based administrative healthcare database. Scand J Public Health 2007; 35: 424-31. doi: 10.1080/14034940701195230

12. Carlsson AC, Wandell P, Osby U, Zarrinkoub R, Wettermark B, Ljunggren G. High prevalence of diagnosis of diabetes, depression, anxiety, hypertension, asthma and COPD in the total population of Stockholm, Sweden - a challenge for public health. BMC Public Health 2013; 13: 670. doi: 10.1186/1471-2458-13-670

13. Ettehad D, Emdin CA, Kiran A, Rahimi K. Blood pressure lowering for cardiovascular disease - authors' reply. Lancet (London, England) 2016; 388: 126-7. doi: 10.1016/S0140-6736(16)30669-9

14. Farah R, Zeidan RK, Chahine MN, Asmar R, Chahine R, Salameh P, et al. Predictors of uncontrolled blood pressure in treated hypertensive individuals: first population-based study in Lebanon. J Clin Hypertens (Greenwich) 2016; 18: 871-7. doi: 10.1111/jch.12775

15. Knight EL, Bohn RL, Wang PS, Glynn RJ, Mogun H, Avorn J. Predictors of uncontrolled hypertension in ambulatory patients. Hypertension 2001; 38: 809-14. doi: 10.1161/hy0901.091681

16. Mallat SG, Samra SA, Younes F, Sawaya MT. Identifying predictors of blood pressure control in the Lebanese population - a national, multicentric survey - I-Predict. BMC Public Health 2014; 14: 1142. doi: 10.1186/1471-2458-14-1142

17. Morgado M, Rolo S, Macedo AF, Pereira L, Castelo-Branco M. Predictors of uncontrolled hypertension and antihypertensive medication nonadherence. J Cardiovasc Dis Res 2010; 1: 196-202. doi: 10.4103/ 0975-3583.74263

18. Shelley D, Tseng TY, Andrews H, Ravenell J, Wu D, Ferrari P, et al. Predictors of blood pressure control among hypertensives in community health centers. Am J Hypertens 2011; 24: 1318-23. doi: 10.1038/ajh.2011.154 
19. Pickering TG, Shimbo D, Haas D. Ambulatory blood-pressure monitoring. N Engl J Med 2006; 354: 2368-74. doi: 10.1056/NEJMra060433

20. Redon J, Lurbe E. Ambulatory blood pressure monitoring is ready to replace clinic blood pressure in the diagnosis of hypertension: con side of the argument. Hypertension 2014; 64: 1169-74; discussion 74. doi: 10.1161/HYPERTENSIONAHA.114.03883

21. Schwan A. Reference values for 24 -hour non-invasive ambulatory blood pressure: a population study of men aged fifty. Scand J Prim Health Care 1993; 11: 21-5. doi: 10.3109/02813439308994897

22. Banegas JR, Ruilope LM, de la Sierra A, de la Cruz JJ, Gorostidi M, Segura J, et al. High prevalence of masked uncontrolled hypertension in people with treated hypertension. Eur Heart J 2014; 35: 3304-12. doi: 10.1093/ eurheartj/ehu016

23. Head GA. Ambulatory blood pressure monitoring is ready to replace clinic blood pressure in the diagnosis of hypertension: pro side of the argument. Hypertension 2014; 64: 1175-81; discussion 81. doi: 10.1161/ HYPERTENSIONAHA.114.03882

24. Banegas JR, Ruilope LM, de la Sierra A, Vinyoles E, Gorostidi M, de la Cruz JJ, et al. Relationship between clinic and ambulatory blood-pressure measurements and mortality. N Engl J Med 2018; 378: 1509-20. doi: 10.1056/NEJMoa1712231

25. Niiranen TJ, Maki J, Puukka P, Karanko H, Jula AM. Office, home, and ambulatory blood pressures as predictors of cardiovascular risk. Hypertension 2014; 64: 281-6. doi: 10.1161/HYPERTENSIONAHA.114.03292

26. Hedberg P, Hammar C, Selmeryd J, Viklund J, Leppert J, Hellberg A, et al. Left ventricular systolic dysfunction in outpatients with peripheral atherosclerotic vascular disease: prevalence and association with location of arterial disease. Eur J Heart Fail 2014; 16: 625-32. doi: 10.1002/ejhf.95

27. Bromfield SG, Booth JN, 3rd, Loop MS, Schwartz JE, Seals SR, Thomas $\mathrm{SJ}$, et al. Evaluating different criteria for defining a complete ambulatory blood pressure monitoring recording: data from the Jackson Heart Study. Blood Press Monit 2018; 23: 103-11. doi: 10.1097/ MBP.0000000000000309

28. Levey AS, Stevens LA, Schmid CH, Zhang YL, Castro AF, 3rd, Feldman HI, et al. A new equation to estimate glomerular filtration rate. Ann Intern Med 2009; 150: 604-12. doi: 10.7326/0003-4819-150-9-200905050-00006

29. De Backer G, Ambrosioni E, Borch-Johnsen K, Brotons C, Cifkova R, Dallongeville J, et al. European guidelines on cardiovascular disease prevention in clinical practice: third joint task force of European and other societies on cardiovascular disease prevention in clinical practice (constituted by representatives of eight societies and by invited experts). Eur Heart J 2003; 24: 1601-10. doi: 10.1016/S0195-668X(03)00347-6
30. Mach F, Baigent C, Catapano AL, Koskinas KC, Casula M, Badimon L, et al. 2019 ESC/EAS Guidelines for the management of dyslipidaemias: lipid modification to reduce cardiovascular risk. Eur Heart J 2020; 41: 111-88. doi: 10.1093/eurheartj/ehz455

31. Barna I, Keszei A, Dunai A. Evaluation of meditech ABPM-04 ambulatory blood pressure measuring device according to the British Hypertension Society protocol. Blood Press Monit 1998; 3: 363-8.

32. O'Brien E, Parati G, Stergiou G, Asmar R, Beilin L, Bilo G, et al. European society of hypertension position paper on ambulatory blood pressure monitoring. J Hypertens 2013; 31: 1731-68. doi: 10.1097/ HJH.0b013e328363e964

33. Skoglund PH, Ostergren J, Svensson P. Ambulatory pulse pressure predicts cardiovascular events in patients with peripheral arterial disease. Blood Press 2012; 21: 227-32. doi: 10.3109/00365599.2012.676755

34. Itoga NK, Tawfik DS, Lee CK, Maruyama S, Leeper NJ, Chang TI. Association of blood pressure measurements with peripheral artery disease events. Circulation 2018; 138: 1805-14. doi: 10.1161/ CIRCULATIONAHA. 118.033348

35. Thomas Manapurathe D, Krishna SM, Dewdney B, Moxon JV, Biros $\mathrm{E}$, Golledge J. Effect of blood pressure lowering medications on leg ischemia in peripheral artery disease patients: a meta-analysis of randomised controlled trials. PLoS One 2017; 12: e0178713. doi: 10.1371/ journal.pone. 0178713

36. Sigvant B, Wiberg-Hedman K, Bergqvist D, Rolandsson O, Wahlberg E. Risk factor profiles and use of cardiovascular drug prevention in women and men with peripheral arterial disease. Eur J Cardiovasc Prev Rehabil 2009; 16: 39-46. doi: 10.1097/HJR.0b013e32831c1383

37. Sigvant $B$, Hasvold $P$, Thuresson $M$, Jernberg $T$, Janzon $M$, Nordanstig J. Myocardial infarction and peripheral arterial disease: treatment patterns and long-term outcome in men and women results from a Swedish nationwide study. Eur J Prev Cardiol 2019: 2047487319893046. doi: $10.1177 / 2047487319893046$

38. Kumbhani DJ, Steg PG, Cannon CP, Eagle KA, Smith SC, Jr., Goto S, et al. Statin therapy and long-term adverse limb outcomes in patients with peripheral artery disease: insights from the REACH registry. Eur Heart J 2014; 35: 2864-72. doi: 10.1093/eurheartj/ehu080

39. Stock JK. The challenge of peripheral arterial disease: how do we improve outcome? Atherosclerosis 2018; 270: 196-8. doi: 10.1016/j. atherosclerosis.2017.12.031

40. Sjahid SI, van der Linden PD, Stricker BH. Agreement between the pharmacy medication history and patient interview for cardiovascular drugs: the Rotterdam elderly study. Br J Clin Pharmacol 1998; 45: 591-5. doi: 10.1046/j.1365-2125.1998.00716.x 